\title{
Asymmetry of parental origin in long QT syndrome: preferential maternal transmission of $K C N Q 1$ variants linked to channel dysfunction
}

\author{
Hideki Itoh ${ }^{\star, 1,2,3}$, Myriam Berthet ${ }^{1,2,4}$, Véronique Fressart ${ }^{1,2,4,5}$, Isabelle Denjoy ${ }^{1,6}$, Svetlana Maugenre ${ }^{1,2,4}$, \\ Didier Klug ${ }^{7}$, Yuka Mizusawa ${ }^{8}$, Takeru Makiyama ${ }^{9}$, Nynke Hofman ${ }^{10}$, Birgit Stallmeyer ${ }^{11}$, Sven Zumhagen ${ }^{11}$, \\ Wataru Shimizu ${ }^{12,13}$, Arthur AM Wilde ${ }^{8}$, Eric Schulze-Bahr ${ }^{11,14}$, Minoru Horie ${ }^{3}$, Sophie Tezenas du Montcel ${ }^{15,16}$ \\ and Pascale Guicheney ${ }^{1,2,4}$
}

Transmission distortion of disease-causing alleles in long QT syndrome (LQTS) has been reported, suggesting a potential role of $K C N Q 1$ and $K C N H 2$ in reproduction. This study sought to investigate parental transmission in LQTS families according to ethnicity, gene loci (LQT1-3: KCNQ1, KCNH2, and SCN5A) or severity of channel dysfunction. We studied 3782 genotyped members from 679 European and Japanese LQTS families (2748 carriers). We determined grandparental and parental origins of variant alleles in 1903 children and 624 grandchildren, and the grandparental origin of normal alleles in healthy children from 44 three-generation control families. LQTS alleles were more of maternal than paternal origin (61 vs $39 \%, P<0.001)$. The ratio of maternally transmitted alleles in LQT1 $(66 \%)$ was higher than in LQT2 $(56 \%, P<0.001)$ and LQT3 $(57 \%, P=0.03)$. Unlike the Mendelian distribution of grandparental alleles seen in control families, variant grandparental LQT1 and LQT2 alleles in grandchildren showed an excess of maternally transmitted grandmother alleles. For LQT1, maternal transmission differs according to the variant level of dysfunction with $68 \%$ of maternal transmission for dominant negative or unknown functional consequence variants vs $58 \%$ for non-dominant negative and variants leading to haploinsufficiency, $P<0.01$; however, for LQT2 or LQT3 this association was not significant. An excess of disease-causing alleles of maternal origin, most pronounced in LQT1, was consistently found across ethnic groups. This observation does not seem to be linked to an imbalance in transmission of the LQTS subtype-specific grandparental allele, but to the potential degree of potassium channel dysfunction.

European Journal of Human Genetics (2016) 24, 1160-1166; doi:10.1038/ejhg.2015.257; published online 16 December 2015

\section{INTRODUCTION}

Long QT syndrome (LQTS) is an inherited arrhythmogenic syndrome with QT interval prolongation, syncope, and risk of sudden death from polymorphic ventricular tachycardia or ventricular fibrillation. Thirteen candidate genes are implicated, of which two potassium channel genes (KCNQ1, LQT1 [MIM 192500] and KCNH2, LQT2 [MIM 613688]) and a sodium channel gene (SCN5A, LQT3 [MIM 603830]) are the major genetic subtypes, accounting for $\sim 80 \%$ of all genotyped LQTS patients. ${ }^{1}$

We previously reported transmission ratio distortion (TRD) in comprehensively genotyped European LQTS families, with an excess of variant carriers and female predominance. ${ }^{2}$ TRD is defined as a significant deviation from expected Mendelian ratios of allele inheritance, that is, a preferential transmission of a variant allele of particular parental origin. In consequence, this may lead to an unexpected higher frequency of disease in offspring. The mechanisms underlying TRD in LQTS are unclear. Whereas siblings should randomly receive grandparent origin alleles, Naumova $e t \mathrm{al}^{3}$ reported grandparental transmission distortion in normal subjects for imprinted regions such as the 11 p15.5 locus, where KCNQ1 is located. They suggested that the TRD in LQT1 could be linked to a locus-specific distortion over three-generation transmission. ${ }^{4}$ Accordingly, in this study we compared the grandparental origin of normal alleles in control families from the Centre d'Étude du Polymorphisme Humain (CEPH) with that of variant alleles in LQTS families.

On the other hand, several studies have demonstrated the expression of KCNQ1 voltage-gated potassium channels in the ovaries, in granulosa and trophoblastic cells. ${ }^{5-7}$ Thus, the voltagegated potassium channel may have an important role in zygote maturation.

${ }^{1}$ INSERM, UMR S1166, Paris, France; ${ }^{2}$ Sorbonne Universités, UPMC Univ Paris 06, UMR S1166, Paris, France; ${ }^{3}$ Department of Cardiovascular and Respiratory Medicine, Shiga University of Medical Science, Otsu, Japan; ${ }^{4}$ Institute of Cardiometabolism and Nutrition, ICAN, Pitié-Salpêtrière Hospital, Paris, France; ${ }^{5}$ AP-HP, Groupe Hospitalier PitiéSalpétrière, Service de Biochimie Métabolique, UF Cardiogénétique et Myogénétique Moléculaire et Cellulaire, Paris, France; ${ }^{6}$ Cardiology Department, AP-HP, Hôpital Bichat, Paris, France; ${ }^{7}$ Hôpital Cardiologique de Lille, CHRU, Service de cardiologie A, Lille, France; ${ }^{8}$ AMC Heart Center, Department of Clinical and Experimental Cardiology, Academic Medical Center, University of Amsterdam, Amsterdam, The Netherlands; ${ }^{9}$ Department of Cardiovascular Medicine, Kyoto University Graduate School of Medicine, Kyoto, Japan; ${ }^{10}$ Department of Clinical Genetics, Academic Medical Center, University of Amsterdam, Amsterdam, The Netherlands; ${ }^{11}$ Department of Cardiovascular Medicine, Institute for Genetics of Heart Diseases (IfGH), University Hospital Münster, Münster, Germany; ${ }^{12}$ Division of Arrhythmia and Electrophysiology, Department of Cardiovascular Medicine, National Cerebral and Cardiovascular Center, Suita, Japan; ${ }^{13}$ Division of Cardiology, Department of Internal Medicine, Nippon Medical School, Tokyo, Japan; ${ }^{14}$ Interdisciplinary Centre for Clinical Research (IZKF) of the University of Münster, Münster, Germany; ${ }^{15}$ Biostatistics Unit, AP-HP, Groupe Hospitalier Pitié-Salpêtrière Charles-Foix, Paris, France; ${ }^{16}$ Sorbonne Universités, UPMC Univ Paris 06, INSERM, Institut Pierre Louis d’Epidémiologie et de Santé Publique (IPLESP UMRS 1136), Paris, France

${ }^{*}$ Correspondence: Dr H Itoh, Department of Cardiovascular and Respiratory Medicine, Shiga University of Medical Science, Seta Tsukinowa-cho, Shiga, Otsu 520-2192, Japan. Fax: +81 77543 5839; E-mail: itohhide@belle.shiga-med.ac.jp

Received 29 March 2015; revised 30 October 2015; accepted 15 November 2015; published online 16 December 2015 
We followed maternal and paternal transmission of LQT1-LQT3 variant alleles in a large number of families from Europe (France, Germany and the Netherlands) and Asia (Japan), and explored the link between parental origin asymmetry and the severity of channel dysfunction.

\section{MATERIALS AND METHODS Subjects}

Genotyped LQT1-LQT3 families with known disease-causing variants, of at least two generations, were collected from three European centers (Paris, France; Münster, Germany; and Amsterdam, The Netherlands) and three Japanese centers (Shiga University of Medical Science, Kyoto University Graduate School of Medicine, and Suita National Cerebral and Cardiovascular Center). Genotyping in each center followed written informed consent and the ethical rules of each country. The subjects included in the study were as follows: LQTS patients carrying one variant, obligate carriers with or without documented cardiac sudden death, and non-carriers who were descendants of LQTS patients. If both parents carried a LQTS variant, their descendants were excluded. The cohort comprised 679 families (2748 carriers and 1034 non-carriers), of which 164 European families were analyzed previously ${ }^{2}$ and 515 families were new additions, which included the entire Japanese cohort. The variants have been submitted to the Leiden Open Variation Database (LOVD 3.0, www.LOVD.nl/KCNQ1, KCNH2, and SCN5A) with IDs given in Supplementary Tables S1 and S2 (LQT1, 49 181-49 512; LQT2, 49 515-49 793; and LQT3, 47 336-47 343, except 47 341, 47 469-47 495, and 49 027-49 060). A total of 83 sudden cardiac deaths occurred before the age of 40 years, 44 in LQT1 (19 male and 25 female), 28 in LQT2 (7 male, 21 female), and 11 in LQT3 ( 8 male and 3 female). The cases who died but not from sudden death were 8 females and 2 males.

\section{Origin of the variant alleles}

We determined the parental origin of the allele transmitted to proband and all carriers from each family without regard to phenotype. When Mendelian transmission is respected, variant alleles are transmitted from mothers and fathers in a 1:1 ratio. Furthermore, we evaluated the grandparental origin of variant alleles in three-generation LQTS families and the grandparental transmission of KCNQ1, KCNH2, and SCN5A loci markers in threegeneration control families, to determine whether grandparental transmission distortion occurs. We then compared the grandparental origin of LQTS variant alleles with that of normal alleles. Figure 1 shows allele transmission in a large LQT1 family.

\section{Genotypes in control families}

We studied 44 three-generation CEPH families, including the 31 families analyzed by Naumova et $a^{\beta}$, and 13 additional kindreds (1353, 1354, 1356, $1357,1358,1447,1451,1454,1458,1459,1463,1477$, and 1582). Markers within or near each of the genes were genotyped: D11S4088 and D11S1318 for KCNQ1, D7S2461 for KCNH2, and D3S1260 and D3S3521 for SCN5A. For some families, we also used data from the CEPH genotype database as shown in Supplementary Figure S1. Haplotypes allowed us to determine the grandparental origin for 2025 alleles including $712 \mathrm{KCNQ1}$ alleles, $703 \mathrm{KCNH} 2$ alleles, and 610 SCN5A alleles (Supplementary Tables S3-S5). Statistical analyses were performed on the 31 families in Naumowa et $a b^{3}$ on all the families, and after exclusion of some families when the origin of the four grandparental alleles could not be determined (for KCNQ1 locus, see Supplementary Table S6). Three families were excluded for KCNQ1, four for $\mathrm{KCNH} 2$, and eight for SCN5A.

\section{Variant categories}

To determine whether transmission distortion was linked to channel dysfunction, we classified $K C N Q 1$ variants according to location and type. ${ }^{8}$ For channel
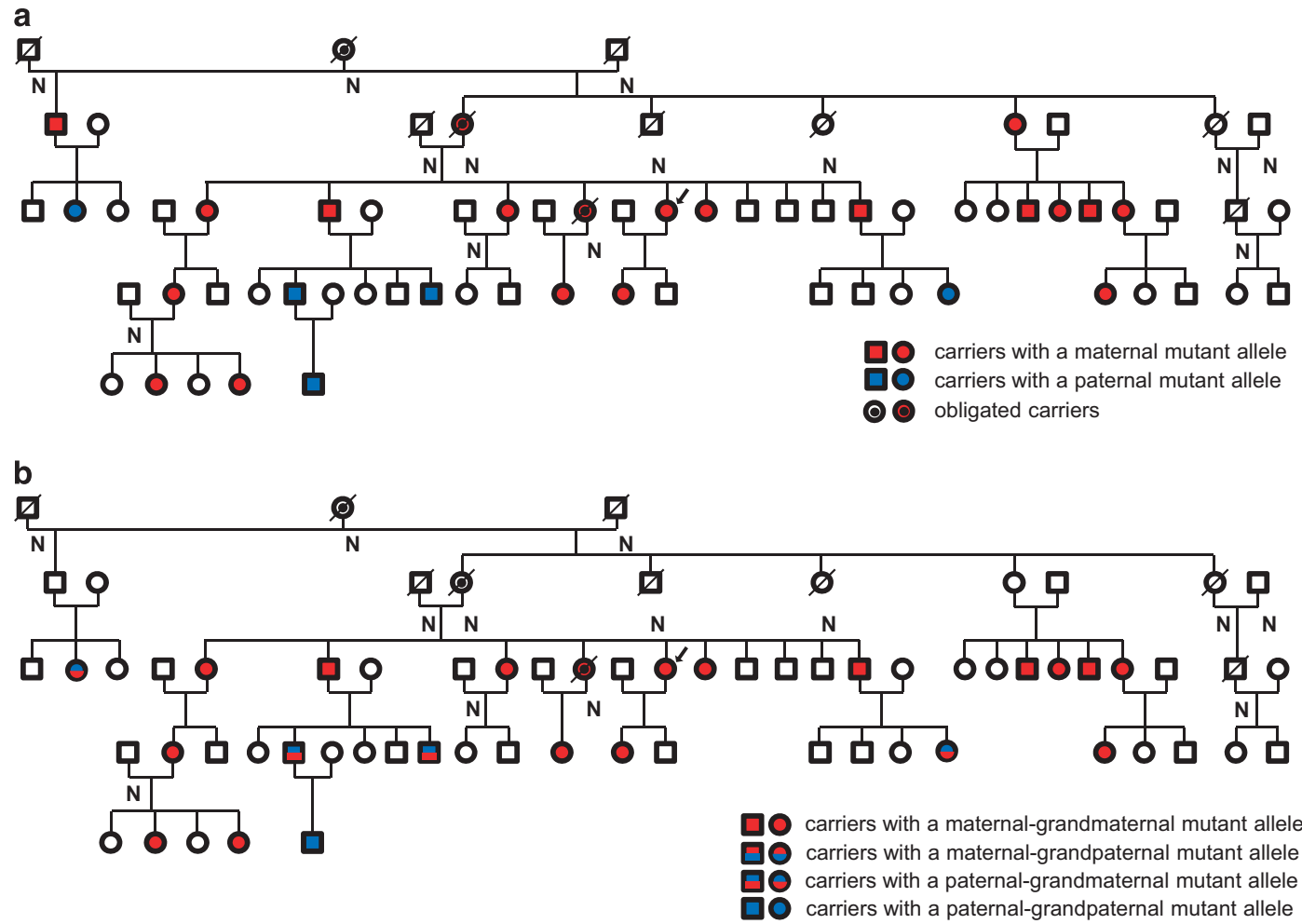

Figure 1 Parental and grandparental origin of variant alleles in a LQT1 family. In this pedigree, there are 25 carriers (18 women) of the R555C variant including three obligate carriers. The classification of the variant carriers is illustrated by colored symbols. (a) Parental origin of LQTS alleles transmitted to carriers. (b) Grandparental origin of the LQTS alleles transmitted to the carriers. N, not tested. Proband is indicated by an arrow, and open circles and squares are non-carriers. 
dysfunction, missense variants were categorized according to either a dominant ( $>50 \%$ reduction in $I_{\mathrm{Ks}}$ current; $n=24$ ) or a non-dominant negative effect $(n=9)$ on wild-type subunits, according to published heterologous expression studies. Other non-missense variants such as nonsense $(n=6)$, splice site $(n=7)$, and frameshift variants $(n=22)$ with known or assumed loss of function were grouped as haploinsufficiency variants (Supplementary Table S1). The last group comprises mostly variants with unknown function including many missense $(n=76)$, some splice site $(n=7)$, and in-frame deletion variants $(n=7)$.

For $\mathrm{KCNH} 2$, variants of the S5-pore-S6 caused the most severe phenotypes, mostly due to a dominant negative effect, ${ }^{9}$ and we therefore categorized $\mathrm{KCNH} 2$ variants into four classes: N-terminus, S1-S4, S5-pore-S6, and C-terminus (Supplementary Table S2). We also classified LQT2 variants according to variant type, as for LQT1. We did not analyze LQT3 variants, as the size of the group did not allow subdivisions and there is no evidence of risk stratification for $S C N 5 A$ variants.

\section{Statistical analysis}

Differences between two groups were analyzed by the $\chi^{2}$-test or Fisher's exact test where appropriate. Two-tailed exact binomial tests were used to assess whether the ratio of parental or grandparental alleles differed from 0.5 or 0.25 , respectively. The odds ratios (ORs) with $95 \%$ confidence interval (CI) were estimated as a measure of association. Statistical significance was considered as $P<0.05$. Ages at sudden death were compared using an unpaired Student's $t$-test.

\section{RESULTS}

Table 1 shows analysis of the genotyped cohort according to ethnicity, LQTS genes, carrier or non-carrier status, and gender, demonstrating female predominance among LQTS carriers (1563 LQTS females of 2748, 57 vs 43\% males) but not among non-carriers where the sex distribution was nearly equal (524 females of 1034, 51 vs 49\% males; $P=0.0004)$. This was due to a larger number of female carriers in LQT1 and LQT2, but not in LQT3 families. The results were similar in both Japanese and European samples.

\section{Preferential maternal transmission of LQTS alleles}

Among the 479 LQTS probands, $61 \%$ inherited the variant from their mothers (maternal vs paternal: 292 alleles $v s 187$ alleles, $P<0.0001$ ).
When all the carriers were considered, we observed the same asymmetry, with $61 \%$ alleles of maternal origin (1155 of 1892 variant alleles, $P<0.0001)$. Sixty-six percent of LQT1 carriers received the variant from their mothers (602 of 910 carriers, $P<0.0001$ ), highly significant for children of both genders for the whole cohort, and in the subgroups from France, Germany, and Japan, but not from the Netherlands (Figure 2 and Supplementary Table S7). The preferential maternal transmission was less impressive for LQT2 carriers and for LQT3 carriers, but still significant (LQT2: $56 \%, P=0.0008$ and LQT3: $57 \%, P=0.036$ ), mostly due to a preferential transmission to female offspring (Supplementary Table S7). This results in a genotype-specific significantly larger maternal variant allele ratio among LQT1 carriers than for LQT2 and LQT3 (OR: 1.5, 95\% CI: $1.3-1.9, P<0.0001$ and OR: $1.5,95 \%$ CI: $1.1-2.0, P=0.011)$.

\section{Grandparental-allele transmission in CEPH and LQTS families}

We determined the grandparental origin of alleles transmitted to grandchildren for the three LQTS genes in CEPH families. The allele ratios from each grandparent varied from 0.23 to 0.27 , not significantly different from the expected value of 0.25 (Figure 3 and Supplementary Table S8). In contrast, comparison of grandparental LQTS allele ratios to control alleles for each locus, showed significant distortion for allele transmission in three generation LQT1 and LQT2 families (LQT1, $P<0.0001$ and LQT2, $P<0.025$, but not in LQT3 families $(P=0.25$; Figure 3$)$. In LQT1, transmission to grandchildren of both genders was distorted, with an excess of maternal grandmother alleles (39\%) and a reduction of paternal grandfather alleles $(13 \%$; $P<0.0001)$, whereas in LQT2 families a significant discordant transmission was only found for female grandchildren compared with that for CEPH families ( $P=0.036$; Supplementary Table S8).

\section{Parental origin asymmetry and variant classification}

We aimed to determine whether the observed distortion was dependent on ion channel dysfunction. We thus classified LQT1 variants into subgroups according to type, location, and their functional effect, (i) either leading to haploinsufficiency, (ii) with a proven dominant negative or (iii) non-dominant negative effect on

Table 1 Cohort according to LQTS genes, gender and countries

\begin{tabular}{|c|c|c|c|c|c|c|c|c|c|c|c|c|c|c|c|c|}
\hline & \multicolumn{4}{|c|}{ Total } & \multicolumn{3}{|c|}{ France } & \multicolumn{3}{|c|}{ Germany } & \multicolumn{3}{|c|}{ The Netherlands } & \multicolumn{3}{|c|}{ Japan } \\
\hline & $\mathrm{n}$ & $(\%)$ & P-value & $\mathrm{P}$-value ${ }^{\mathrm{b}}$ & $\mathrm{n}$ & $(\%)$ & $\mathrm{P}$-value & $\mathrm{n}$ & $(\%)$ & P-value & $\mathrm{n}$ & $(\%)$ & P-value & $\mathrm{n}$ & $(\%)$ & P-value \\
\hline \multicolumn{17}{|l|}{ Families } \\
\hline All & 679 & & & & 199 & & & 147 & & & 85 & & & 248 & & \\
\hline LQT1 & 332 & & & & 126 & & & 74 & & & 19 & & & 113 & & \\
\hline LQT2 & 279 & & & & 68 & & & 66 & & & 34 & & & 111 & & \\
\hline LQT3 & 68 & & & & 5 & & & 7 & & & 32 & & & 24 & & \\
\hline \multicolumn{17}{|c|}{ Carriers female/all (\% of female) } \\
\hline All & $1563 / 2748$ & $(57)$ & & & $487 / 871$ & $(56)$ & & $373 / 650$ & $(57)$ & & $301 / 557$ & (54) & & $402 / 670$ & $(60)$ & \\
\hline LQT1 & $763 / 1314$ & (58) & $<0.0001$ & 0.0045 & $317 / 559$ & $(57)$ & 0.0015 & $178 / 308$ & (58) & 0.0062 & $72 / 133$ & (54) & 0.34 & $196 / 314$ & $(62)$ & $<0.0001$ \\
\hline LQT2 & $647 / 1135$ & $(57)$ & $<0.0001$ & 0.066 & $158 / 286$ & (55) & 0.076 & $174 / 311$ & $(56)$ & 0.037 & $141 / 247$ & $(57)$ & 0.026 & $174 / 291$ & $(60)$ & 0.0008 \\
\hline LQT3 & $153 / 299$ & $(51)$ & 0.68 & 0.47 & $12 / 26$ & (46) & 0.84 & $21 / 31$ & (68) & 0.07 & $88 / 177$ & $(50)$ & 0.9 & $32 / 65$ & (49) & 0.94 \\
\hline \multicolumn{17}{|c|}{ Non-carriers female/all (\% of female) } \\
\hline All & $524 / 1034$ & $(51)$ & & & $215 / 404$ & (53) & & $138 / 258$ & (53) & & $119 / 259$ & $(46)$ & $52 / 113$ & (46) & & \\
\hline LQT1 & $208 / 415$ & (50) & 0.96 & & $112 / 229$ & (49) & 0.74 & $55 / 87$ & (63) & 0.013 & $18 / 45$ & $(40)$ & 0.27 & $23 / 54$ & (43) & 0.17 \\
\hline LQT2 & $253 / 486$ & (52) & 0.36 & & $97 / 165$ & (59) & 0.024 & $78 / 158$ & (49) & 0.87 & $55 / 116$ & $(47)$ & 0.88 & $23 / 47$ & (49) & 0.57 \\
\hline LQT3 & 63/133 & $(47)$ & 0.54 & & $6 / 10$ & (60) & 0.75 & $5 / 13$ & (38) & 0.58 & 46/98 & $(47)$ & 1 & $6 / 12$ & (50) & 0.54 \\
\hline
\end{tabular}

${ }^{a} \chi^{2}$-Test when compared with a female ratio assumed to be of 0.5 .

b $x^{2}$-Test when compared with female ratios in non-carriers. 
wild-type alleles, or (iv) with unknown functional consequences (Figure 4 and Supplementary Figure S2). Interestingly, variants leading to haploinsufficiency such as splice, nonsense, frameshift variants, and missense variants with non-dominant negative effects, which both cause the mildest channel dysfunction, were found associated with less distortion (58\% maternal transmission) compared with dominant-

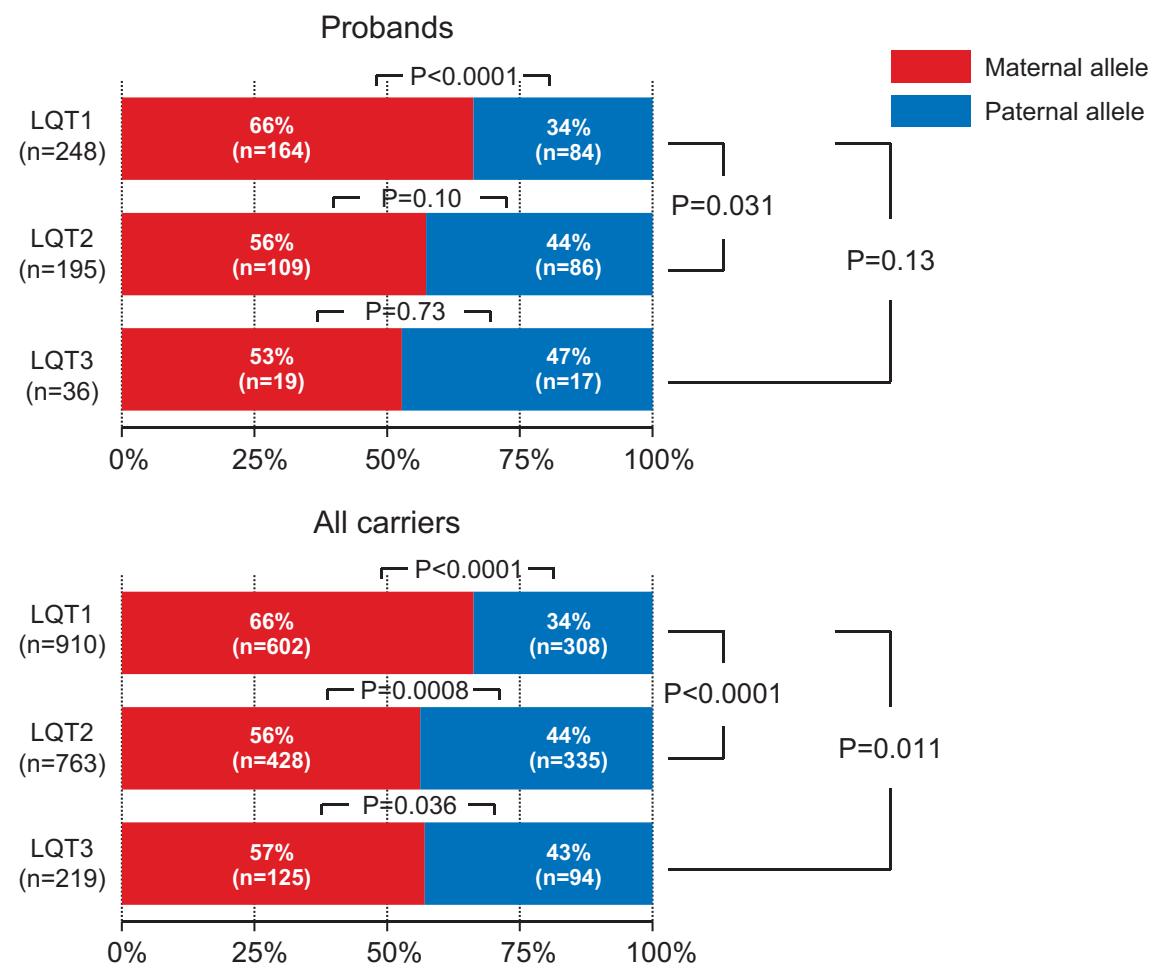

Figure 2 Parental origin of variant alleles transmitted to probands or all carriers for each LQTS subtype.

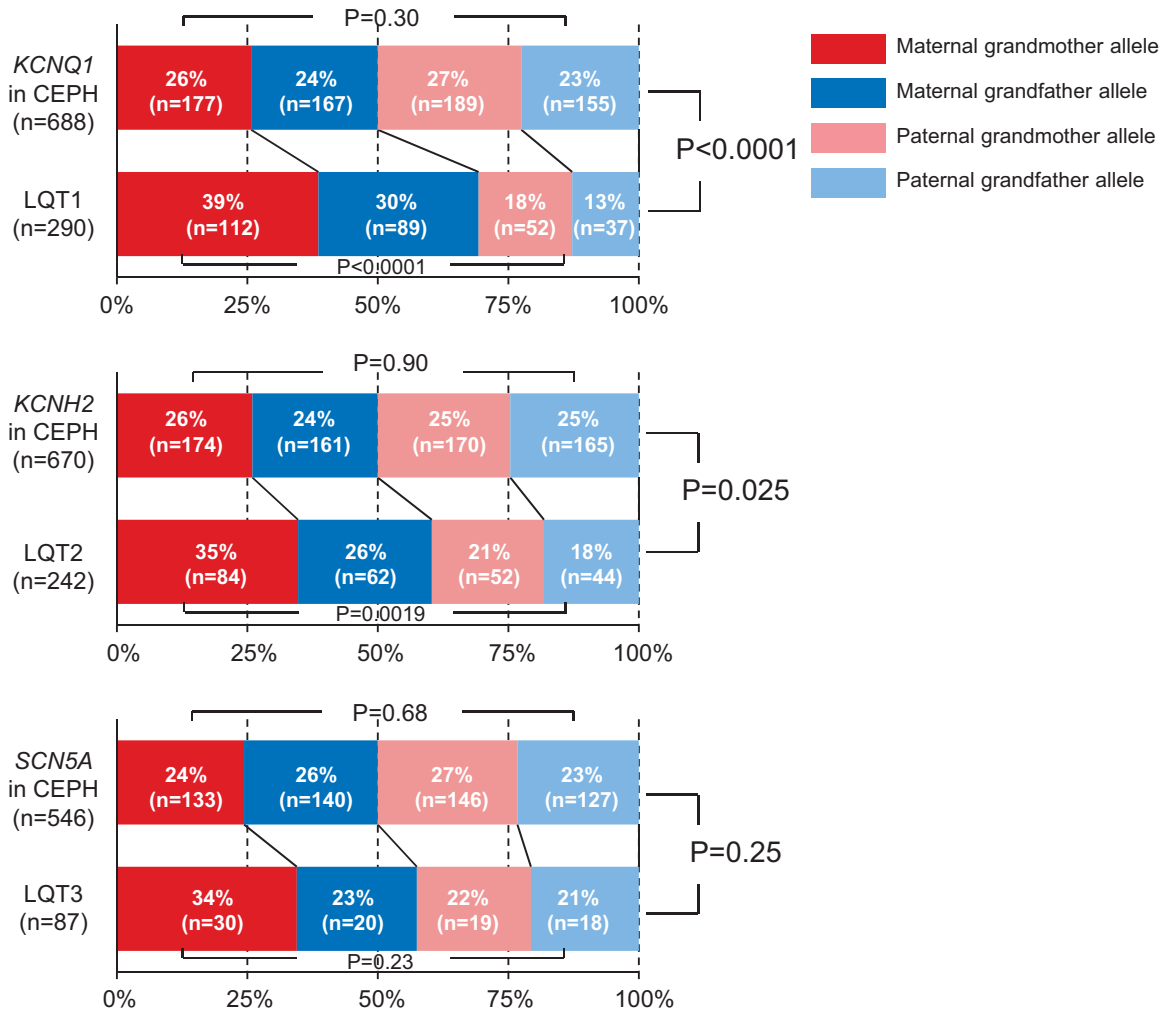

Figure 3 Grandparental origins of LQTS alleles for each LQT subtype. The upper bar shows the allele distribution of the four grandparental origins in CEPH families and the lower bar shows the variant-allele distribution of four grandparental origins in LQTS. 


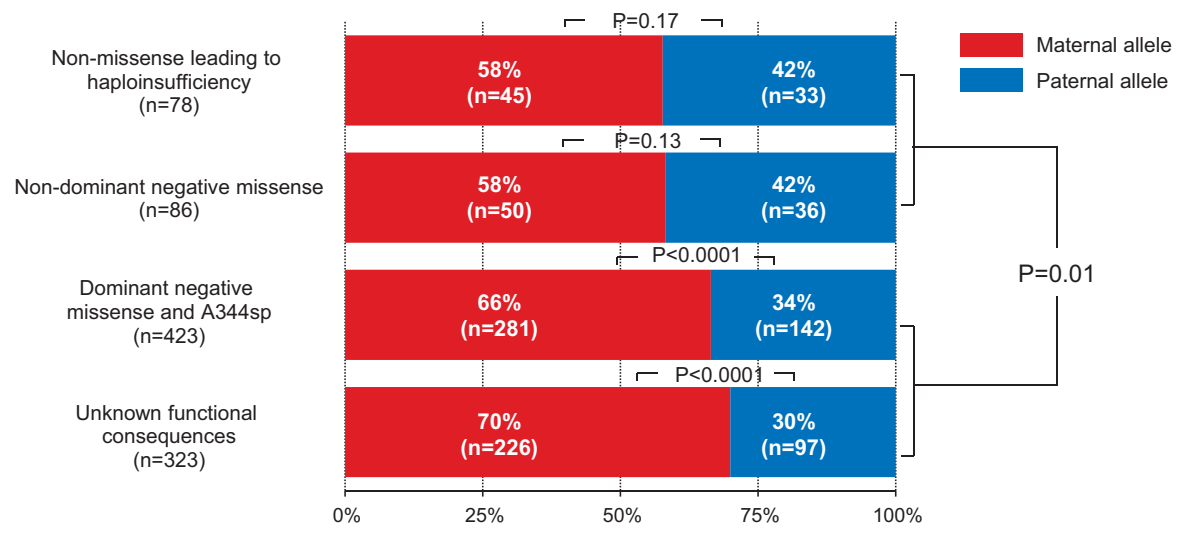

Figure 4 Parental origins of LQT1 alleles and channel dysfunction. LQT1 variants were classified as non-missense variants, leading to haploinsufficiency, non-dominant negative missense variants, and dominant negative missense variants when their co-expression with wild-type channels induced $>50 \%$ reduction in $I_{\text {Ks }}$ current according to published patch clamp analyses. The last group corresponds to variants with unknown functional consequences. The maternal transmission compared between the four function groups under the assumption that the four maternal transmission are equal to 0.5 was significantly different $(P<0.0001)$. For more explanations on classification, see Supplementary Table S1.

negative missense variants, and with other variants, a heterogeneous group, which mostly includes missense variants with unknown effects (68\%, $P=0.01)$.

For LQT2, maternal transmission was higher than the paternal transmission only for missense variants (58 vs $42 \%, P=0.002$ ). A similar distortion was observed for missense variants located in the pore region of the protein (S5-pore-S6) compared with other missense variants (Supplementary Figure S3).

\section{DISCUSSION}

This study provides information on parental and grandparental transmission in a large number of genotyped LQTS patients from different countries. We report an asymmetrical parental origin for variant LQTS alleles, with higher-than-expected maternal transmission and inheritance, especially for LQT1, in both European and Japanese families. In addition to this, we found that this is not linked to a LQTS subtype (locus-specific) grandparental origin allele transmission distortion, but probably associated with the severity of channel dysfunction. This suggests that the altered function of the potassium channel, especially KCNQ1, could modulate reproduction.

We had previously reported transmission distortion in LQTS families, demonstrating female predominance and an excess of carriers through segregation transmission in LQT1 and LQT2 families. ${ }^{2}$ Our data also suggested a tendency to more frequent transmission of LQTS alleles from mothers to daughters. Furthermore, Naumova et $a^{3}$ reported in 2001 an asymmetrical transmission of the imprinted chromosome 11 p15.5 region through meiosis in $31 \mathrm{CEPH}$ families, with a significantly higher transmission rate of paternal grandfather alleles to sons and of maternal grandmother alleles to daughters. They suggested that grandparental origin-dependent transmission distortion is related to imprinting, an epigenetic factor that could be responsible for transmission distortion of several genes, ${ }^{3,10}$ including KCNQ1, and thus could explain transmission distortion in LQT1. ${ }^{4}$ To study this hypothesis, we compared grandparental allele transmission in $\mathrm{CEPH}$ families for the LQT1-3 gene loci. We differed on two methodological points compared with the Naumova study: we studied more families and used highly polymorphic intragenic markers or markers more closely linked to the loci of interest. We found that the grandparental allele distribution was normal for the three loci whether we included all families or only those in which the four grandparental alleles were traced. This is concordant with a study on the Framingham heart cohort, which does not report distortion for chromosome $11 .{ }^{11}$ If we did not observe significant TRD for the KCNQ1 locus throughout the three generations in control families, this does not exclude a TRD for other loci on 11p15.5, such as INS-IGF2 locus, which is distant of $300 \mathrm{~kb}^{12}$

In contrast, distribution analysis of grandparental variant alleles to grandchildren in LQTS families showed an obvious and significant excess of maternal grandmother LQTS alleles in LQT1 and LQT2 families, and a net reduction of paternal grandfather LQTS alleles, compared with values expected from Mendelian laws and CEPH family ratios. For LQT3 families, an increased ratio of grandmaternal alleles cannot be formally excluded; however, the variations did not reach significance, owing to the limited number of cases. The marked distortion observed in grandparental LQTS allele transmission correlates well with the more frequent transmission of maternal LQT1 or LQT2 alleles in nuclear families, and results from a cumulative effect during two generations.

The mechanism resulting in the higher transmission rate of maternal LQTS alleles, highly significant in LQT1 families, is not known. A reduction in the number of male carriers of reproductive age, compared with female carriers, could induce female predominance and higher maternal transmission. However, in LQTS, even if gender differences have been demonstrated, they do not support this theory. At adult age, LQT1 and LQT2 women have longer QT intervals and a higher risk of life-threatening cardiac events than men. ${ }^{13,14}$ By contrast, before puberty, the incidence of arrhythmia is higher and occurred at a younger age in boys than in girls, ${ }^{15}$ but the numbers of sudden deaths are similar between men and women LQTS patients in most of the studies, as it was in our cohort, where even more females than males died from non-arrythmogenic causes. Over several decades, $\beta$-blocker therapy has been used to prevent sudden death in LQTS patients, and intrauterine or infancy deaths due to LQTS remained exceptional. ${ }^{16}$ Even if it is unlikely that premature death in males could explain the sex imbalance and the transmission distortion among patients in our population, we cannot exclude that fetal wastage of embryos could occur at very early stage of development, as $50-75 \%$ of all human conceptions are lost before the first missed menses. ${ }^{17,18}$ It is known that abnormal imprint resetting could lead to early death of embryos ${ }^{19}$ and KCNQ1 is a paternally imprinted gene. Nevertheless, it is difficult to conceive the specific loss of male $v s$ female embryos. 
In contrast to other imprinted genes, for which only one of the two parental alleles is expressed in tissues during all the life, KCNQ1 paternal imprint is reversed during development, and both maternal and paternal alleles could be detected in most of the tissues. This was shown in mouse $\mathrm{s}^{20}$ and a partial erasing was reported in tissues from human embryos, especially in hearts. ${ }^{21}$ To our knowledge, no other study was performed to determine whether the KCNQ1 paternal imprint is fully erased or not in all human tissues. Nevertheless, it is clear that the cardiac phenotype of LQT1 sibs are similar regardless of the gender of parents with a variant in KCNQ1 as shown for the Japanese cohort (Supplementary Table S9). This suggests a normal expression of both alleles in the heart.

We rechecked in the enlarged pool of families, the transmission to fully genotyped offspring applying correction for single ascertainment ${ }^{22}$ as in our earlier study. ${ }^{2}$ Our previous observation in LQT1 families that an excess of carriers is only present in descendants from affected mothers, but not from affected fathers, was confirmed (Supplementary Table S10). We postulate that the normal transmission of paternal LQT1 variants is probably due to the repression of paternal KCNQ1 allele expression by imprinting in spermatozoids and early embryogenesis. By comparison, in LQT2 and LQT3 families, there was also an excess of carriers but without significant difference between maternal and paternal transmissions; these genes are not in imprinted loci. The excess of maternal variant alleles in LQT1 was thus probably related to the sole expression of the maternal alleles and suggested that the LQT1 variants may confer a sex-differential reproduction rate. Following this hypothesis, our objective was to determine whether the degree of ion channel dysfunction could influence preferential allele transmission, and this was clearly observed for LQT1 variants. We found a less pronounced distortion for the heterozygous variants recognized to be associated with the mildest dysfunction. This subgroup consisted of variants leading to haploinsufficiency such as nonsense, frameshift, and splice variants. Conversely, we found a maternal transmission rate of $67 \%$ for the dominant negative variants, which are associated with the most severe channel dysfunction, compared with $58 \%$ for non-dominant negative variants. $^{8}$

Thus, the degree of transmission distortion in LQTS may vary among populations depending on variant type. This may explain the absence of significant female predominance/transmission distortion recently reported in a Norwegian population where most of the identified LQT1 variants led to haploinsufficiency and a moderate channel dysfunction. ${ }^{23}$

Nevertheless, there is no obvious link between the increased maternal transmission and the female predominance, these two observations may result from different mechanisms involving oocytes, spermatozoids, or other maternal tissues. As KCNQ1 is expressed in numerous organs and in different types of cells, variants that alter myocardial repolarization might also have important effects in other tissues and at early stages of development such as fertilization in which ion channels are crucially important. ${ }^{24}$

Differential co-assembly of KCNQ1 with single transmembrane $K C N E \beta$-subunits in different cell types gives rise to a variety of biophysical properties, thus endowing distinct physiological roles for KCNQ1-KCNEx complexes. ${ }^{6,20,25}$ Some of them are well-known. In the heart, KCNQ1 co-assembles with KCNE1, the major cardiac $\beta$-subunit, to form the slowly activating $\mathrm{K}+$ channel sensitive to adrenergic regulation. ${ }^{8,26}$ In contrast, co-assembly of KCNQ1 with KCNE3 produces a current with nearly instantaneous activation. ${ }^{27}$ In colonic crypts, these KCNQ1:KCNE3 channels mediate basolateral $\mathrm{K}^{+}$recycling required for $\mathrm{Cl}^{-}$secretion and their expression is regulated by estrogens. ${ }^{28}$ In the stomach, KCNQ1:KCNE2 regulates acid secretion. ${ }^{29}$ Interestingly, KCNQ1 is also expressed in porcine and human granulosa cells, as well as in large follicles where it could have a role in follicular maturation. ${ }^{5,30}$ In the ovaries, acetylcholine elevates intracellular calcium levels, thereby opening a calcium-activated potassium channel (BKCa), an action resulting in membrane hyperpolarization. ${ }^{31}$ This latter event could allow activation of other voltage-dependent ion channels, which are linked to steroid production. ${ }^{32}$ Besides, KCNQ1 is regulated by acetylcholine, a novel intraovarian signaling molecule. ${ }^{5}$ In cultured granulosa cells, inhibition of $\mathrm{K}^{+}$channels by a KCNQ channel blocker, XE991, inhibited gonadotropin-stimulated steroid production and increased cell proliferation. ${ }^{5}$ This suggests a crucial role for KCNQ channels in the follicle. The effect of a KCNQ1-specific blocker has not been studied so far; however, if it also increases granulosa cell proliferation, this may contribute to the maturation of oocytes carrying a LQT1 variant, and thus the maternal transmission and the observed TRD in favor of carriers in the families.

Classification of $\mathrm{KCNH} 2$ variants based on dysfunction remains difficult, as many missense variants affect to a variable level channel trafficking and expression when others impair biophysical properties. ${ }^{33,34}$ We found more maternal than paternal transmission of LQT2 variants but the differences were smaller than for LQT1 variants. The ratios were of 0.58 for missense variants and 0.54 for variants leading to haploinsufficiency, and a similar distortion was observed for missense variants located in the pore region of the protein (S5-pore-S6) compared with other missense variants. The $\mathrm{KCNH} 2$ channels are developmentally regulated and may also have a role in early mouse embryogenic development. ${ }^{35}$

It is noteworthy that although we postulate that the loss-of-function of the cardiac potassium channels could induce transmission distortion and contribute to female predominance in LQTS, a marked male predominance, up to $80 \%$, has been observed among patients with a short QT syndrome. ${ }^{36,37}$ This syndrome is caused by gain-of-function variants in the same channels where loss-of-function variants lead to LQTS, but only in a limited number of cases. It is tempting to suggest a possible role for channel dysfunction in this male predominance, but this must be tempered by the fact that most of the genetic background of this syndrome has not been elucidated.

Further studies on larger cohorts are needed to determine whether gain- or loss-of-function SCN5A variants could also influence parental transmission and to elucidate the origin of the preferential maternal transmission of KCNQ1 and $\mathrm{KCNH} 2$ variants.

\section{Study limitations}

This study is a retrospective study and has several limitations. The variability in size of the families could be a limitation. Large families were collected to study allele transmission across three generations, but small families were also included, especially those from Japan. It is interesting to note that female predominance among carriers and preferential maternal transmission were both observed in this Asian population for LQT1 and LQT2. Most of the LQT3 families, generating a smaller cohort than for LQT1 and LQT2, were from the Netherlands and harbored the same founder variant, c.5385_5387dup (p.(Tyr1795_Glu1796insAsp)). The Netherlands LQT1 and LQT2 cohorts were too small to show true differences from those of the other countries.

\section{CONCLUSIONS}

From our multi-national cohort of LQTS families, there was an excess of grandmaternal and maternal variant alleles with the strongest effect 
for LQT1. We favor the hypothesis that the dysfunction of potassium channels could have a major role in preferential maternal transmission in LQTS.

\section{CONFLICT OF INTEREST}

The authors declare no conflict of interest.

\section{ACKNOWLEDGEMENTS}

We are grateful to Dr Graham Macdonald and late Professor Josué Feingold for helpful discussions; Dr Megumi Fukuyama for the collection of Japanese LQTS families; Dr Sven Zumhagen and Dr Birgit Stallmeyer for support in German LQTS family sampling; and Dr Rachel Peat for article proofing. ES-B was supported by the Fondation Leducq, Paris, and the DFG (Schu1082/3-1 and 3-2) and the Interdisciplinary Center for Clinical Research (IZKF; Schu1/011/12) of the Medical Faculty of the University of Münster. This work is also supported by a Grant-in-Aid for Scientific Research from JSPS (HI).

1 Cerrone M, Priori SG: Genetics of sudden death: focus on inherited channelopathies Eur Heart J 2011; 32: 2109-2118.

2 Imboden M, Swan H, Denjoy I et al: Female predominance and transmission distortion in the long-QT syndrome. N Engl J Med 2006; 355: 2744-2751.

3 Naumova AK, Greenwood CM, Morgan K: Imprinting and deviation from Mendelian transmission ratios. Genome 2001; 44: 311-320.

4 Naumova AK: Long-QT syndrome. N Engl J Med 2007; 356: 1680, author reply 1680.

5 Kunz L, Roggors C, Mayerhofer A: Ovarian acetylcholine and ovarian KCNQ channels: insights into cellular regulatory systems of steroidogenic granulosa cells. Life Sci 2007; 80: 2195-2198.

6 Mistry HD, McCallum LA, Kurlak LO, Greenwood IA, Broughton Pipkin F, Tribe RM: Novel expression and regulation of voltage-dependent potassium channels in placentas from women with preeclampsia. Hypertension 2011; 58: 497-504.

7 Luo Y, Kumar P, Mendelson CR: Estrogen-related receptor $\gamma$ (ERR $\gamma$ ) regulates oxygendependent expression of voltage-gated potassium $(\mathrm{K}+)$ channels and tissue kallikrein during human trophoblast differentiation. Mol Endocrinol 2013; 27: 940-952.

8 Moss AJ, Shimizu W, Wilde AA et al: Clinical aspects of type-1 long-QT syndrome by location, coding type, and biophysical function of mutations involving the KCNQ1 gene. Circulation 2007; 115: 2481-2489.

9 January CT, Gong Q, Zhou Z: Long QT syndrome: cellular basis and arrhythmia mechanism in LQT2. J Cardiovasc Electrophysiol 2000; 11: 1413-1418.

10 Dean NL, Loredo-Osti JC, Fujiwara TM et al: Transmission ratio distortion in the myotonic dystrophy locus in human preimplantation embryos. Eur J Hum Genet 2006; 14: 299-306.

11 Liu Y, Zhang L, Xu S, Hu L, Hurst LD, Kong X: Identification of two maternal transmission ratio distortion loci in pedigrees of the Framingham heart study. Sci Rep 2013; 3: 2147

12 Eaves IA, Bennett ST, Forster $\mathrm{P}$ et al: Transmission ratio distortion at the INSIGF2 VNTR. Nat Genet 1999; 22: 324-325.

13 Sauer AJ, Moss AJ, McNitt S et al: Long QT syndrome in adults. J Am Coll Cardiol 2007; 49: 329-337.
14 Conrath CE, Wilde AA, Jongbloed RJ et al: Gender differences in the long QT syndrome: effects of beta-adrenoceptor blockade. Cardiovasc Res 2002; 53: 770-776.

15 Locati EH, Zareba W, Moss AJ et al: Age- and sex-related differences in clinical manifestations in patients with congenital long-QT syndrome: findings from the International LQTS Registry. Circulation 1998; 97: 2237-2244.

16 Crotti L, Tester DJ, White WM et al: Long QT syndrome-associated mutations in intrauterine fetal death. JAMA 2013; 309: 1473-1482.

17 Edmonds DK, Lindsay KS, Miller JF, Williamson E, Wood PJ: Early embryonic mortality in women. Fertil Steril 1982; 38: 447-453.

18 Rushton DI SJ, Jenkins DM, Goodhart CB: Letter: Where have all the conceptions gone? Lancet 1975; 1: 636-637.

19 Huang LO, Labbe A, Infante-Rivard C: Transmission ratio distortion: review of concept and implications for genetic association studies. Hum Genet 2013; 132: 245-263.

20 Gould TD, Pfeifer K: Imprinting of mouse Kvlqt1 is developmentally regulated. Hum Mol Genet 1998; 7: 483-487.

21 Lee MP, Hu RJ, Johnson LA, Feinberg AP: Human KVLQT1 gene shows tissue-specific imprinting and encompasses Beckwith-Wiedemann syndrome chromosomal rearrangements. Nat Genet 1997; 15: 181-185.

22 Li C: Human Genetics: Principles and Methods. McGraw-Hill: New York, 1961.

23 Berge KE, Haugaa KH, Früh A et al: Molecular genetic analysis of long QT syndrome in Norway indicating a high prevalence of heterozygous mutation carriers. Scand J Clin Lab Invest 2008; 68: 362-368.

24 Shukla KK, Mahdi AA, Rajender S: Ion channels in sperm physiology and male fertility and infertility. J Androl 2012; 33: 777-788.

25 Lundquist AL, Turner CL, Ballester LY, George AL: Expression and transcriptional control of human KCNE genes. Genomics 2006; 87: 119-128.

26 Goldenberg I, Thottathil P, Lopes CM et al: Trigger-specific ion-channel mechanisms, risk factors, and response to therapy in type 1 long QT syndrome. Heart Rhythm 2012; 9: 49-56.

27 Schroeder BC, Waldegger S, Fehr S et al: A constitutively open potassium channel formed by KCNQ1 and KCNE3. Nature 2000; 403: 196-199.

28 Alzamora R, O'Mahony F, Bustos V et al: Sexual dimorphism and oestrogen regulation of KCNE3 expression modulates the functional properties of $\mathrm{KCNQ} \mathrm{K}^{+}$channels. J Physiol 2011; 589: 5091-5107.

29 Grahammer F, Herling AW, Lang HJ et al: The cardiac K+ channel KCNQ1 is essential for gastric acid secretion. Gastroenterology 2001; 120: 1363-1371.

30 Mason DE, Mitchell KE, Li Y, Finley MR, Freeman LC: Molecular basis of voltage-dependent potassium currents in porcine granulosa cells. Mol Pharmacol 2002; 61: 201-213.

31 Kunz L, Thalhammer A, Berg FD et al: Ca2+-activated, large conductance K+ channel in the ovary: identification, characterization, and functional involvement in steroidogenesis. J Clin Endocrinol Metab 2002; 87: 5566-5574.

32 Kunz L, Richter JS, Mayerhofer A: The adenosine 5'-triphosphate-sensitive potassium channel in endocrine cells of the human ovary: role in membrane potential generation and steroidogenesis. J Clin Endocrinol Metab 2006; 91: 1950-1955.

33 Shimizu W, Moss AJ, Wilde AA et al: Genotype-phenotype aspects of type 2 long QT syndrome. J Am Coll Cardiol 2009; 54: 2052-2062.

34 Anderson CL, Delisle BP, Anson BD et al: Most LQT2 mutations reduce Kv11.1 (hERG) current by a class 2 (trafficking-deficient) mechanism. Circulation 2006; 113: 365-373.

35 Winston NJ, Johnson MH, McConnell JM, Cook DI, Day ML: Expression and role of the ether-à-go-go-related (MERG1A) potassium-channel protein during preimplantation mouse development. Biol Reprod 2004; 70: 1070-1079.

36 Giustetto C, Schimpf R, Mazzanti A et al: Long-term follow-up of patients with short QT syndrome. J Am Coll Cardiol 2011; 58: 587-595.

37 Mazzanti A, Kanthan A, Monteforte $\mathrm{N}$ et al: Novel insight into the natural history of short QT syndrome. J Am Coll Cardiol 2014; 63: 1300-1308.

Supplementary Information accompanies this paper on European Journal of Human Genetics website (http://www.nature.com/ejhg) 